\title{
Correction to: Thermalization and Canonical Typicality in Translation-Invariant Quantum Lattice Systems
}

\author{
Markus P. Müller ${ }^{1,2}$, Emily Adlam ${ }^{3}$, Lluís Masanes ${ }^{4}$, Nathan Wiebe ${ }^{5,6}$ \\ 1 Institute for Quantum Optics and Quantum Information, Austrian Academy of Sciences, Boltzmanngasse \\ 3, A-1090 Vienna, Austria. \\ E-mail: markus.mueller@oeaw.ac.at \\ 2 Perimeter Institute for Theoretical Physics, 31 Caroline Street North, Waterloo, ON N2L 2Y5, Canada \\ 3 Centre for Quantum Information and Foundations, DAMTP, Centre for Mathematical Sciences, University \\ of Cambridge, Wilberforce Road, Cambridge, CB3 0WA, UK \\ 4 Department of Computer Science, University College London, Gower Street, London, WC1E 6BT, UK \\ 5 Department of Physics, University of Washington, Seattle, WA 98195, USA \\ 6 Pacific Northwest National Laboratory, Richland, WA 99354, USA
}

Published online: 28 March 2021 - C Springer-Verlag GmbH Germany, part of Springer Nature 2021

Correction to: Commun. Math. Phys. 340, 499-561 (2015) https://doi.org/10.1007/s00220-015-2473-y

In our result about dynamical thermalization, the proof of the upper bound on the time average of the distance between the local evolved state $\rho^{(n)}(t)$ and the time-averaged state $\rho_{\text {avg }}^{(n)}$ is wrong. While it is correct that this distance tends to zero for block size $\left|\Lambda_{n}\right| \rightarrow \infty$ (see corrected proof below), it is unclear whether it can be shown that this happens exponentially fast in $\left|\Lambda_{n}\right|$. This affects Theorem 31, and hence also Theorem 3 (the summary of Theorem 31) and Theorem 33 (a small modification of Theorem 31).

This mistake is due to an error in Ref. [3] which we have used in our proof of Lemma 30. Ref. [3] claims that the Rényi entropy $H_{q}$ is convex in its parameter $q$, which is incorrect. This claim has been corrected in an erratum published on the author's homepage [4], but we became aware of this only recently.

We give a corrected version of Theorem 31 of our paper [1] in Theorem 4 below. Its summary (and hence the correction of Theorem 3 of our paper) reads as follows.

Theorem 1 (Correction of [1, Theorem 3]). If there is a unique equilibrium state around inverse temperature $\beta:=\lim _{n \rightarrow \infty} \beta_{n}$, if the (possibly pure) initial state has close to maximal population entropy, in the sense that

$$
\bar{S}\left(\rho_{0}^{(n)}\right) \geq S\left(\gamma_{\Lambda_{n}}^{p}\left(\beta_{n}\right)\right)-o\left(\left|\Lambda_{n}\right|\right)
$$


and if each $H_{\Lambda_{n}}^{p}$ is non-degenerate with uniformly bounded gap degeneracy $\sup _{n} D_{G}$ $\left(H_{\Lambda_{n}}^{p}\right)<\infty$, then unitary time evolution thermalizes the subsystem $\Lambda$ for most times $t$ :

$$
\left\langle\left\|\operatorname{Tr}_{\Lambda_{n} \backslash \Lambda} \rho^{(n)}(t)-\operatorname{Tr}_{\Lambda_{n} \backslash \Lambda} \frac{\exp \left(-\beta_{n} H_{\Lambda_{n}}^{p}\right)}{Z_{n}}\right\|_{1}\right\rangle \stackrel{n \rightarrow \infty}{\longrightarrow} 0 .
$$

The gap degeneracy [5] is defined as $D_{G}\left(H_{\Lambda_{n}}^{p}\right):=\max _{E} \mid\left\{(i, j) \mid i \neq j, E_{i}-E_{j}=\right.$ $E\} \mid$, with $E_{i}$ the eigenvalues of $H_{\Lambda_{n}}^{p}$.

This formulation differs from the old one in the following two ways. First, it does not give concrete bounds on the time-averaged distance between $\rho^{(n)}(t)$ and its time average (it only says that this distance tends to zero for $n \rightarrow \infty$ ); second, it presumes that the gap degeneracy is uniformly bounded.

To prove its formal version (Theorem 4 below), we need two elementary lemmas.

Lemma 2. Let $\Phi$ be a translation-invariant finite-range interaction which is not physically equivalent to zero, and let $\bar{u}$ be some energy density for which there is a unique Gibbs state at inverse temperature $\beta(\bar{u})$. Then the real function $u \mapsto s(u)$ defined in [1, Lemma 9] is strictly concave at $\bar{u}$ in the following sense: If $\bar{u}=\lambda u_{0}+(1-\lambda) u_{1}$ for some $u_{0}<u_{1}$ and $\lambda \in(0,1)$ then $s(\bar{u})>\lambda s\left(u_{0}\right)+(1-\lambda) s\left(u_{1}\right)$.

Proof. Let $u_{0}<u_{1}$ and $u=\lambda u_{0}+(1-\lambda) u_{1}$ for some $\lambda \in(0,1)$. Let $\omega_{\beta\left(u_{0}\right)}$ be an arbitrary Gibbs state with energy density $u_{0}$ at inverse temperature $\beta\left(u_{0}\right)$, and similarly $\omega_{\beta\left(u_{1}\right)}$. Set $\omega:=\lambda \omega_{\beta\left(u_{0}\right)}+(1-\lambda) \omega_{\beta\left(u_{1}\right)}$, a translation-invariant state. Since the entropy density is affine on the translation-invariant states ([2, Thm. IV.2.4]), we have

$$
s(\omega)=\lambda s\left(\omega_{\beta\left(u_{0}\right)}\right)+(1-\lambda) s\left(\omega_{\beta\left(u_{1}\right)}\right)=\lambda s\left(u_{0}\right)+(1-\lambda) s\left(u_{1}\right) .
$$

By construction, $u(\omega)=u$. Thus, due to [1, Lemma 9], we have $s(\omega) \leq s(u)$, hence $u \mapsto s(u)$ is concave.

Let us now apply the previous argumentation to the special case $u:=\bar{u}$, an energy density with a unique Gibbs state. Suppose that $s(\bar{u})=s(\omega)$. Then the variational principle ([1, Definition 6]) implies that $\omega$ is a Gibbs state at inverse temperature $\beta(\bar{u})$. But the set of Gibbs states at inverse temperature $\beta(\bar{u})$ is a face of the set of all translationinvariant states [2, p. 348], hence $\omega_{\beta\left(u_{0}\right)}$ and $\omega_{\beta\left(u_{1}\right)}$ must both be Gibbs states at inverse temperature $\beta(\bar{u})$, too. But these are distinct states, since they have different energy densities, contradicting the uniqueness of the Gibbs state at $\beta(\bar{u})$. Therefore $s(\bar{u})>s(\omega)$, and we get the statement of strict concavity as claimed.

Lemma 3. Let $\Phi$ be a translation-invariant finite-range interaction which is not physically equivalent to zero. Suppose that the maximal energy degeneracy of $H_{\Lambda_{n}}^{p}$ grows at most subexponentially in $\left|\Lambda_{n}\right|$, i.e. $\log \max \left\{\operatorname{tr}\left(\pi_{i}^{(n)}\right)\right\}=o\left(\left|\Lambda_{n}\right|\right)$, where $\left(\pi_{i}^{(n)}\right)_{i}$ denotes the eigenprojectors of $H_{\Lambda_{n}}^{p}$. Let $\left(\rho^{(n)}\right)_{n \in \mathbb{N}}$ be any sequence of $\Lambda_{n}$-translation-invariant states with

$\left[\rho^{(n)}, H_{\Lambda_{n}}^{p}\right]=0, \quad S\left(\rho^{(n)}\right) \geq s \cdot\left|\Lambda_{n}\right|+o\left(\left|\Lambda_{n}\right|\right), \quad \operatorname{tr}\left(\rho^{(n)} H_{\Lambda_{n}}^{p}\right)=u \cdot\left|\Lambda_{n}\right|+o\left(\left|\Lambda_{n}\right|\right)$,

where $u \in\left(u_{\min }(\Phi), u_{\max }(\Phi)\right)$ is an energy density such that there is a unique Gibbs state at inverse temperature $\beta(u)$, and $s=s(u)$. Then $\max _{i} \operatorname{tr}\left(\rho^{(n)} \pi_{i}^{(n)}\right) \stackrel{n \rightarrow \infty}{\longrightarrow} 0$. 
Proof. We can write $u$ as some convex combination of two distinct energy densities in a small neighborhood of $u$, and then Lemma 2 implies that $s=s(u)>0$. Let us now argue by contradiction. Suppose that $\lambda^{(n)}:=\max _{i} \operatorname{tr}\left(\rho^{(n)} \pi_{i}^{(n)}\right)$ does not converge to zero. Decompose the state $\rho^{(n)}$ as follows:

$$
\rho^{(n)}=\lambda^{(n)} \tau^{(n)}+\left(1-\lambda^{(n)}\right) \sigma^{(n)},
$$

where $\tau^{(n)}=\pi_{i}^{(n)} \rho^{(n)} \pi_{i}^{(n)} / \lambda^{(n)}$ (note that $\lambda^{(n)}>0$ ), with $\pi_{i}^{(n)}$ the maximizing projector. If $\lambda^{(n)} \neq 1$, define $\sigma^{(n)}:=\bar{\pi}_{i}^{(n)} \rho^{(n)} \bar{\pi}_{i}^{(n)} /\left(1-\lambda^{(n)}\right)$, where $\bar{\pi}_{i}^{(n)}:=\mathbf{1}-\pi_{i}^{(n)}$; if $\lambda^{(n)}=1$, set $\sigma^{(n)}=\bar{\pi}_{i}^{(n)} / \operatorname{tr}\left(\bar{\pi}_{i}^{(n)}\right)$ (if $n$ is large enough, then $\pi_{i}^{(n)} \neq \mathbf{1}$, hence this is well-defined). It follows that $\tau^{(n)}$ and $\sigma^{(n)}$ are mutually orthogonal $\Lambda_{n}$-translation-invariant states that commute with $H_{\Lambda_{n}}^{p}$.

The sequences of real numbers $S\left(\sigma^{(n)}\right) /\left|\Lambda_{n}\right|, \operatorname{tr}\left(\sigma^{(n)} H_{\Lambda_{n}}^{p}\right) /\left|\Lambda_{n}\right|, \operatorname{tr}\left(\tau^{(n)} H_{\Lambda_{n}}^{p}\right) /\left|\Lambda_{n}\right|$ and $\lambda^{(n)}$ are all bounded (the latter sequence bounded away from zero by assumption). Thus, we can find a subsequence $\left(n_{k}\right)_{k \in \mathbb{N}}$ such that

$$
\begin{aligned}
& \lambda^{\left(n_{k}\right)} \stackrel{k \rightarrow \infty}{\longrightarrow} \delta>0, \quad \frac{1}{\left|\Lambda_{n_{k}}\right|} S\left(\sigma^{\left(n_{k}\right)}\right) \stackrel{k \rightarrow \infty}{\longrightarrow} s_{1}, \frac{1}{\left|\Lambda_{n_{k}}\right|} \operatorname{tr}\left(\tau^{\left(n_{k}\right)} H_{\Lambda_{n_{k}}}^{p}\right) \stackrel{k \rightarrow \infty}{\longrightarrow} u_{0}, \\
& \frac{1}{\left|\Lambda_{n_{k}}\right|} \operatorname{tr}\left(\sigma^{\left(n_{k}\right)} H_{\Lambda_{n_{k}}}^{p}\right) \stackrel{k \rightarrow \infty}{\longrightarrow} u_{1},
\end{aligned}
$$

where $s_{1}, u_{0}, u_{1}$ are real numbers, and $0<\delta \leq 1$. Due to (1), computing the von Neumann entropy, we have $S\left(\rho^{\left(n_{k}\right)}\right)=\lambda^{\left(n_{k}\right)} S\left(\tau^{\left(n_{k}\right)}\right)+\left(1-\lambda^{\left(n_{k}\right)}\right) S\left(\sigma^{\left(n_{k}\right)}\right)+\mathcal{O}(1)$. Since $S\left(\tau^{\left(n_{k}\right)}\right) \leq \log \operatorname{tr}\left(\pi_{i}^{\left(n_{k}\right)}\right)=o\left(\left|\Lambda_{n_{k}}\right|\right)$, this implies $s \leq(1-\delta) s_{1}$. Thus, $s>0$ yields $\delta<1$. Similarly, computing the energy expectation value, we obtain $u=\delta u_{0}+(1-\delta) u_{1}$.

Suppose that $s_{1} \geq s\left(u_{1}\right)$, then $s_{1}-\beta u_{1} \geq p(\beta, \Phi)$ for $\beta:=\beta\left(u_{1}\right)$, hence [1, Lemma $8]$ implies that we must have equality, i.e. $s_{1}=s\left(u_{1}\right)$. In summary, we conclude that $s_{1} \leq s\left(u_{1}\right)$. Therefore

$$
s(u)=s \leq(1-\delta) s_{1} \leq \delta s\left(u_{0}\right)+(1-\delta) s\left(u_{1}\right) .
$$

Since $s$ is strictly concave at $u$ due to Lemma 2 above, this is only possible if $u_{0}=u_{1}=u$. Hence

$$
0<s(u) \leq(1-\delta) s_{1} \leq(1-\delta) s\left(u_{1}\right)=(1-\delta) s(u)
$$

which is a contradiction.

This allows us to obtain a corrected version of [1, Theorem 31].

Theorem 4 (Correction of [1, Theorem 31]: Thermalization, periodic boundary conditions). Let $\Phi$ be a translation-invariant finite-range interaction which is not physically equivalent to zero. Suppose that the maximal energy degeneracy of $H_{\Lambda_{n}}^{p}$ grows at most subexponentially in $\left|\Lambda_{n}\right|$, i.e. $\log \max \left\{\operatorname{tr}\left(\pi_{i}^{(n)}\right)\right\}=o\left(\left|\Lambda_{n}\right|\right)$, where $\left(\pi_{i}^{(n)}\right)_{i}$ denotes the eigenprojectors of $H_{\Lambda_{n}}^{p}$, and $\sup _{n} D_{G}\left(H_{\Lambda_{n}}^{p}\right)<\infty$. Let $\left(\rho_{0}^{(n)}\right)_{n \in \mathbb{N}}$ be some sequence of initial states on $\Lambda_{n}$ which have energy expectation value $U_{n}:=\operatorname{tr}\left(\rho_{0}^{(n)} H_{\Lambda_{n}}^{p}\right)$ with density $U_{n} /\left|\Lambda_{n}\right|$ converging to some value $u \in\left(u_{\min }(\Phi), u_{\max }(\Phi)\right)$ as $n \rightarrow \infty$, such that there is a unique Gibbs state around inverse temperature $\beta(u)$. 
Define the "population entropy" $\bar{S}\left(\rho_{0}^{(n)}\right):=S\left(\lambda_{1}, \ldots, \lambda_{N}\right)$, where $S$ is Shannon entropy, and $\lambda_{i}:=\operatorname{tr}\left(\rho_{0}^{(n)} \pi_{i}^{(n)}\right)$ is the probability that the $i$-th level is populated. Suppose that for every $n$ large enough, either $H_{\Lambda_{n}}^{p}$ is non-degenerate or every $\pi_{i}^{(n)} \rho_{0}^{(n)} \pi_{i}^{(n)}$ is $\Lambda_{n}$-translation-invariant. Then, determine the inverse temperature $\beta_{n}$ for which

$$
\operatorname{tr}\left(H_{\Lambda_{n}}^{p} \gamma_{\Lambda_{n}}^{p}\left(\beta_{n}\right)\right)=U_{n}, \text { where } \gamma_{\Lambda_{n}}^{p}\left(\beta_{n}\right):=\frac{\exp \left(-\beta_{n} H_{\Lambda_{n}}^{p}\right)}{Z_{n}} .
$$

If the initial states have close to maximal population entropy in the sense that

$$
\bar{S}\left(\rho_{0}^{(n)}\right) \geq S\left(\gamma_{\Lambda_{n}}^{p}\left(\beta_{n}\right)\right)-o\left(\left|\Lambda_{n}\right|\right)
$$

then unitary time evolution $\rho^{(n)}(t):=\exp \left(-i t H_{\Lambda_{n}}^{p}\right) \rho_{0}^{(n)} \exp \left(i t H_{\Lambda_{n}}^{p}\right)$ thermalizes the subsystem $\Lambda_{m}$ for most times $t$ :

$$
\lim _{n \rightarrow \infty}\left\langle\left\|\operatorname{Tr}_{\Lambda_{n} \backslash \Lambda_{m}} \rho^{(n)}(t)-\operatorname{Tr}_{\Lambda_{n} \backslash \Lambda_{m}} \frac{\exp \left(-\beta_{n} H_{\Lambda_{n}}^{p}\right)}{Z_{n}}\right\|_{1}\right\rangle=0,
$$

where $Z_{n}=\operatorname{tr}\left(\exp \left(-\beta_{n} H_{\Lambda_{n}}^{p}\right)\right)$, and $\langle\cdot\rangle$ denotes the average over all times $t \geq 0$. Furthermore, in this statement, $\beta_{n}$ can be replaced by $\beta:=\beta(u)$.

Proof. The only ingredient in the proof of [1, Theorem 31] that has to be corrected is the argument that lower-bounds the "effective dimension" $d_{\text {eff }}$. The old proof erroneously claimed that $d_{\text {eff }}$ grows exponentially in $\left|\Lambda_{n}\right|$, but this relied on a wrong claim about the Rényi entropy of Ref. [3]. We now give a simple alternative argument which makes use of the Rényi entropy $S_{\infty}\left(\lambda_{1}, \ldots, \lambda_{N}\right)=-\log \max _{i} \lambda_{i}$ and the inequality $S_{2} \geq S_{\infty}$ [4]. Namely,

$$
d_{\mathrm{eff}}=\exp \left(S_{2}\left(\lambda_{1}, \ldots, \lambda_{N}\right)\right) \geq \exp \left(S_{\infty}\left(\lambda_{1}, \ldots, \lambda_{N}\right)\right)=\left(\max _{i} \lambda_{i}\right)^{-1} \stackrel{n \rightarrow \infty}{\longrightarrow} \infty
$$

according to Lemma 3 above, applied to the sequence of states $\bar{\rho}_{0}^{(n)}=\sum_{i} \pi_{i}^{(n)} \rho_{0}^{(n)} \pi_{i}^{(n)}$. Since we have assumed that the gap degeneracy is uniformly bounded, this is enough to show that $\rho^{(n)}(t)$ is close to its time average for most times $t$ if $n$ is large. The rest of the proof works without modification (note that $\rho\left(\beta_{n}\right)$ should read $\gamma_{\Lambda_{n}}^{p}\left(\beta_{n}\right)$ ).

Finally, [1, Theorem 33] has to be corrected analogously. We omit the obvious details.

Acknowledgement. We are grateful to Henrik Wilming for pointing out the mistake in Lemma 30 of the old version, and for further helpful discussions.

Publisher's Note Springer Nature remains neutral with regard to jurisdictional claims in published maps and institutional affiliations.

\section{References}

1. Müller, M.P., Adlam, E., Masanes, L1, Wiebe, N.: Thermalization and Canonical Typicality in TranslationInvariant Quantum Lattice Systems. Commun. Math. Phys. 340, 499-561 (2015)

2. Simon, B.: The Statistical Mechanics of Lattice Gases, vol. 1. Princeton University Press, Princeton (1993)

3. Życzwkoski, K.: Rényi extrapolation of Shannon entropy. Open Sys. Inf. Dyn. 10, 297-310 (2003)

4. Życzwkoski, K.: Rényi extrapolation of Shannon entropy, corrigendum, http://www.cft.edu.pl/ karol/ pdf/Zy03b.pdf (2005)

5. Short, A.J., Farrelly, T.C.: Quantum equilibration in finite time. New J. Phys. 14, 013063 (2012) 